\title{
EFFECT OF ORIENTATION CORRELATION ON THE ELASTIC CONSTANTS OF POLYCRYSTALLINE MATERIALS
}

\author{
H. KIEWEL ${ }^{1}$, H. J. BUNGE ${ }^{2}$ and L. FRITSCHE ${ }^{1}$ \\ ${ }^{1}$ Institute of Theoretical Physics B, ${ }^{2}$ Department of Physical Metallurgy, \\ Technical University of Clausthal, Germany
}

(Received 2 April 1996)

\begin{abstract}
The macroscopic elastic properties of a polycrystalline aggregate depend on the elastic anisotropy of its crystallites and on the texture. Besides that, also geometrical structure parameters have an influence which is confined within the Voigt- and Reuss-averages as upper and lower bounds. These geometrical parameters are grain sizes, grain shapes, and mutual arrangement of grains e.g. number of neighbours and orientation correlation. In the present paper this latter influence, i.e. orientation correlation was studied by model calculations based on a cluster model described earlier. It was found that "low-anglecorrelation" leads to higher elastic stiffness and "high-angle-correlation" results in elastically "softer" materials compared with the uncorrelated arrangement of the same crystal orientations in the aggregate.
\end{abstract}

KEY WORDS: Elastic constants, orientation correlation, misorientation, cluster method.

\section{INTRODUCTION}

The elastic properties of polycrystalline materials are certain averages of the corresponding properties of the constituting grains. Since in most cases single crystal properties are anisotropic the average properties depend essentially on the crystallographic orientation distribution function of the crystallites i.e. the texture of the material.

The individual grains interact elastically across their boundaries. Thereby boundary conditions for the displacement as well as for the stress have to be fulfilled. This leads to a complicated stress-strain field inside the crystallites. Hence, the macroscopic elastic properties also depend on the sizes, shapes and mutual arrangement of grains in the polycrystalline aggregate, i.e. on structural parameters in addition to the texture which does not take any location parameter into account.

Two extreme forms of aggregates consist in long, parallel rods and in broad plates stacked in normal direction. Approximately these two structures correspond to the Reuss-average of constant stress and the Voigt-average of constant strain, respectively (Voigt (1910), Reuss (1929)). These two averages constitute upper and lower bounds between which the effective polycrystal data lie (Hill, 1952).

Under the constraint that their is no orientation correlation between neighbouring grains Hashin and Shtrikman (1962a, b) obtained narrower bounds.

Finally, the assumption of a statistically completely random aggregate leads to a unique average (Kröner, 1958). Kröner's assumption can also be applied to non-random orientation distributions, i.e. texture as studied by Kneer $(1964,1965)$, Morris (1971), 
keeping, however, the assumption of randomness with respect to all location parameters. The method of Kröner is based on the inclusion problem solved by Eshelby (1957). Eshelby assumed one ellipsoidal grain embedded in a surrounding medium. This same principle can be further generalized by constructing a cluster of several or even many crystallites and fulfilling all boundary conditions between them (Kiewel and Fritsche, 1994). This latter method allows to take a great variety of "location" parameters of polycrystalline aggregates strictly into account. Particularly the following structural parameters were considered in some previous investigations (Kiewel and Fritsche (1994), Kiewel, Bunge and Fritsche (1995, 1996a, 1996b)):

- The axis ratio of parallelepipedic grains.

- Shape and arrangement of "equiaxed" grains (i.e. Wigner-Seitz cells of different cubic lattices).

- Orientation of crystal axes versus cell axes.

In these investigations the different crystal orientations were distributed "at random" over the crystallites, the shapes and locations of which were definitely fixed. This means there was no preferred correlation of orientations across the grain boundaries.

In the present paper we consider the influence of various orientation correlations, i.e. assumptions about preferred neighbourhood relations keeping thereby the texture constant.

\section{ORIENTATION CORRELATION}

We assume that the polycrystalline material consists of a finite number of crystallites forming an aggregate with fixed shape and arrangement of the crystallites specified, for instance, by the network of grain boundaries as shown schematically in Figure 1. The crystallite at the location $\alpha$ has the orientation

$$
g^{(\alpha)}=\left(g_{i j}^{(\alpha)}\right)=\left\{\varphi_{1} \Phi \varphi_{2}\right\}^{(\alpha)}=\{\mathbf{r}, \omega\}^{(\alpha)}
$$

which may be specified by the orientation matrix $\left(g_{i j}^{(\alpha)}\right)$, by Euler angles $\left\{\varphi_{1} \Phi \varphi_{2}\right\}^{(\alpha)}$ or by a rotation axis $\mathbf{r}^{(\alpha)}$ and rotation angle $\omega^{(\alpha)}$. Thereby $g$ is the rotation which brings the sample coordinate system $K_{A}$ into coincidence with the crystal coordinate system $K_{B}$ (see e.g. Bunge (1982)). If the crystals belong to higher than triclinic symmetry then symmetrically equivalent orientations numbered by $n$

$$
g_{n}^{(\alpha)}=g_{n}^{c} \cdot g^{(\alpha)} \quad(1 \leq n \leq N)
$$

with total number $N$ cannot be distinguished. Thereby $g_{n}^{c}$ is one of the crystal symmetry operations i.e. symmetry rotations. (For details see (Bradley and Cracknell, 1972).)

We assign to each grain $\alpha$ its volume $V^{(\alpha)}$. The Orientation Distribution Function (ODF) i.e. the texture of the material is defined by

$$
\frac{1}{V} \sum_{\alpha(g)} V^{(\alpha)}(g)=f(g) d g \quad(\mathrm{ODF})
$$




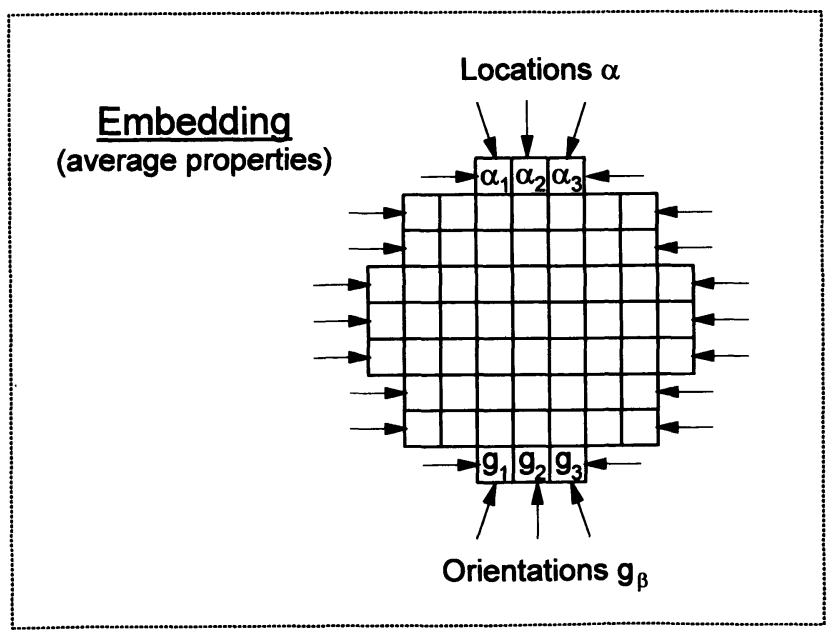

Figure 1 Crystal locations $\alpha$ and orientations $g_{(\beta)}$. The cut is taken through the centre of a cluster consisting of 81 cubes.

where the sum runs over all grains having the orientation $g . V$ denotes the volume of the entire aggregate.

The misorientation $\Delta g^{\left(\alpha^{\prime} \alpha\right)}$ between two crystallites in the locations $\alpha^{\prime}$ and $\alpha$ is

$$
\Delta g^{\left(\alpha^{\prime} \alpha\right)}=g^{\left(\alpha^{\prime}\right)} \cdot\left(g^{(\alpha)}\right)^{-1} .
$$

Similar to Eq. (1) also the rotation $\Delta g^{\left(\alpha^{\prime} \alpha\right)}$ can be represented in the forms

$$
\Delta g^{\left(\alpha^{\prime} \alpha\right)}=\left(\Delta g_{i j}^{\left(\alpha^{\prime} \alpha\right)}\right)=\left\{\varphi_{1} \Phi \varphi_{2}\right\}^{\left(\alpha^{\prime} \alpha\right)}=\{\mathbf{r}, \omega\}^{\left(\alpha^{\prime} \alpha\right)}
$$

In analogy to Eq. (2) the misorientation must be the same when crystal symmetry is applied to either crystal $\alpha^{\prime}$ or $\alpha$

$$
\Delta g_{n_{1} n_{2}}^{\left(\alpha^{\prime} \alpha\right)}=g_{n_{1}}^{c} \cdot \Delta g^{\left(\alpha^{\prime} \alpha\right)} \cdot g_{n_{2}}^{c} \quad\left(1 \leq n_{1}, n_{2} \leq N\right) .
$$

We define analogously to Eq. (3) the Misorientation Distribution Function (MODF) (see e.g. Bunge and Weiland (1988))

$$
\frac{1}{A} \sum_{\left(\alpha^{\prime} \alpha\right)(\Delta g)} A^{\left(\alpha^{\prime} \alpha\right)}(\Delta g)=F(\Delta g) d \Delta g \quad(\mathrm{MODF})
$$

where $A$ denotes the total area of all grain boundaries. The sum runs over all boundaries between grains $\alpha^{\prime}$ and $\alpha$ having the misorientation $\Delta g$. Their areas are designated by $\mathrm{A}^{\left(\alpha^{\prime} \alpha\right)}(\Delta g)$. It is apparent that the MODF, $F(\Delta g)$, is not yet fixed by the ODF, $f(g)$. On the other hand, the MODF is not completely independent of the ODF. Rather it is restricted by the "available" orientations contained in $f(g)$. This influence can be taken into consideration by defining the "uncorrelated MODF" which is the autocorrelation function of the texture 


$$
F_{u}(\Delta g)=\oint f(\Delta g \cdot g) \cdot f(g) d \Delta g
$$

This MODF is to be expected if we distribute the "available" orientations $g$, given by the texture, "at random" over the grain locations $\alpha$. Then the orientation correlation function $C(\Delta g)$ can be defined (Bunge and Weiland, 1988)

$$
C(\Delta g)=\frac{F(\Delta g)}{F_{u}(\Delta g)} \equiv 1 \text { uncorrelated }
$$

We define the "norm" of the rotation $g$ by

$$
|g|=\left[\sum_{i, j}\left(g_{i j}-\delta_{i j}\right)^{2}\right]^{\frac{1}{2}} .
$$

This is related to the rotation angle $\omega$ of Eq. (1) by

$$
\cos \omega=1-\frac{|g|^{2}}{4} .
$$

An analogous definition also applies to the misorientation $\Delta g$ and its rotation angle $\omega$ according to Eq. (5). In a first approximation it follows from Eq. (11) for small angles $\omega$

$$
\omega \approx \frac{|g|}{\sqrt{2}} .
$$

In the case of misorientation $\Delta g^{\left(\alpha^{\prime} \alpha\right)}$ all equivalent misorientations $\Delta g_{n_{1} n_{2}}^{\left(\alpha^{\prime} \alpha\right)}$ must be considered. The norm is taken for that one with the smallest rotation angle $\omega$ which also corresponds to the minimum of $\left|\Delta g_{n_{1} n_{2}}^{\left(\alpha^{\prime} \alpha\right)}\right|$ with respect to all $n_{1}, n_{2}$

$$
\left|\Delta g^{\left(\alpha^{\prime} \alpha\right)}\right|=\min _{n_{1}, n_{2}}\left|\Delta g_{n_{1} n_{2}}^{\left(\alpha^{\prime} \alpha\right)}\right| \text {. }
$$

\section{CONSTRUCTION OF PARTICULAR MODFS}

We now consider crystal orientations $g_{(\beta)}$ which we may distribute in many different ways over the crystal locations $\alpha$. For simplicity, we assume that all crystal locations $\alpha$ have the same volume $V^{(\alpha)}=$ const. as in Figure 1 . Then the orientations $g_{(\beta)}$ define the same texture, which way ever we distribute them over the locations $\alpha$. Each particular distribution defines, however, a particular MODF, Eq. (7). Many different MODFs may be defined according to many different assumptions. For example, we may request that a certain function $E$ of $\Delta g^{\left(\alpha^{\prime} \alpha\right)}$, Eq. (5), takes on an extreme value when averaged over all grain boundaries $\left(\alpha^{\prime} \alpha\right)$ with total number $N_{\left(\alpha^{\prime} \alpha\right)}$

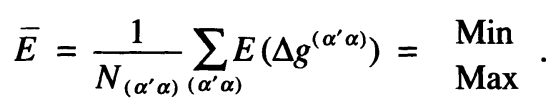


For instance, $E$ may be the grain boundary energy, or it may be the discontinuity of certain components of the elasticity tensor across the grain boundary, or it may be the norm $|\Delta g|$, Eq. (10), or simply the rotation angle $\omega$, Eq. (12). Each of these choices defines a particular MODF.

In order to approximate any so defined MODF a stochastic algorithm was used. At first the chosen orientations $g_{(\beta)}$ were distributed at random over the locations $\alpha$. This leads to the uncorrelated MODF Eq. (8). For simplicity, we describe the algorithm in the following for only two locations $\alpha$ and $\alpha^{\prime}$. The required generalizations become then evident. The locations have the orientations $g_{(\beta)}^{(\alpha)}$ and $g_{\left(\beta^{\prime}\right)}^{\left(\alpha^{\prime}\right)}$ which results in a value $\bar{E}_{1}$ for $\bar{E}$ in Eq. (14). These orientations were exchanged, i.e. they became $g_{\left(\beta^{\prime}\right)}^{(\alpha)}$ and $g_{(\beta)}^{(\alpha)}$. One then obtains the respective value $\bar{E}_{2}$ for $\bar{E}$. If $\bar{E}$ approaches the extremum i.e.

$$
\bar{E}_{2}-\bar{E}_{1}<0
$$

this exchange was carried out, otherwise the original combination was kept. This procedure was repeated many times for the exchange of small numbers of locations until no further improvement of the function $\bar{E}$ towards the assumed extremum could be achieved.

In the present paper we considered particularly the "norm" of the misorientation defined according to Eq. (13). This norm was either minimized or maximized according to Eq. (14).

The algorithm extremizing Eq. (14) depends essentially on the neighbourhoodrelationships of the grain locations $\alpha$. For the sake of simplicity, grain locations were used here which form one of three cubic lattices i.e. the simple cubic (sc), face centred cubic (fcc), and body centred cubic (bcc). This defines the neighbourhood-relationships between the $\alpha$ uniquely. It also defines the shapes of grains as was already studied earlier (Kiewel, Bunge and Fritsche, 1996a).

\section{RESULTS AND DISCUSSION}

Within the three types of cells used here the individual grains are arranged in "shells", i.e. our construction scheme starts with one grain in the centre of the cluster. It is then surrounded by nearest neighbours, next nearest ones and so on.

In the present paper, the orientations $g_{(\beta)}$ were chosen at random i.e. the material is macroscopically isotropic. The function $E$ in Eq. (14) was particularly chosen according to Eq. (13) i.e. the norm of misorientation. The obtained results were, however, plotted in terms of $\omega$ according to Eq. (11).

Figure 2(a) displays distributions of misorientations obtained for sc-clusters consisting of 515 grains. The solid curve shows results for the "original" cluster whose grain orientations were determined by a random generator. If one interchanges these orientations between the individual grains until the sum $E$ of Eq. (14) converges to a minimum one obtains the dotted curve. The dashed curve results if one maximizes $E$. All the curves were smoothed by Gaussians, which were normalized with respect to $|\Delta g|$. As has to be, the average values of the distributions attain a minimum (maximum) if one minimizes (maximizes) $E$ and the half-widths of the respective Gaussians become much smaller. Because of the smoothening of the curves values for $\omega$ can occur with a very small but non-zero probability which are larger 
than the maximum possible value $\omega_{\max }$ for $\omega$. By using elementary relations one can show that $\omega_{\max } \approx 62.80^{\circ}$ for cubic crystal symmetry (Mackenzie, 1964).

Figures 2(b) and (c) show the same results for fec- and bcc-clusters consisting of 321 and 339 grains, respectively. The curves for these clusters are nearly identical in contrast to those for the sc-clusters which show significant deviations from the two

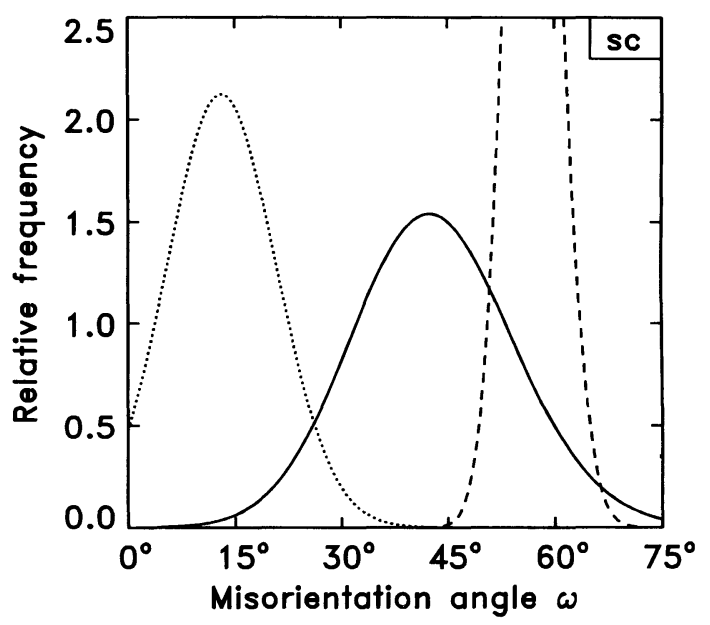

(a)

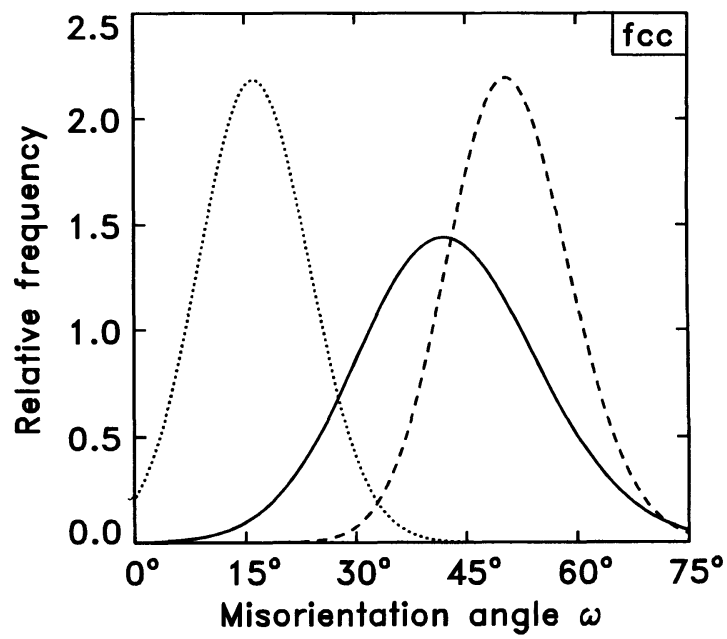

(b)

Figure 2 (a) Distribution of misorientations for three clusters consisting of 515 cubes. The orientation of grains for the first cluster was determined by a random generator (solid curve). The dotted curve was obtained by interchanging the orientations until the sum $E$ of Eq. (14) attains a minimum. The dashed curve results if one maximizes E. All curves were smoothed by Gaussians, which were normalized with respect to $|\Delta g|$. (b) The same situation as in (a) except that we have replaced the cubic grains by 321 Wigner-Seitz cells of a face centred cubic lattice. 


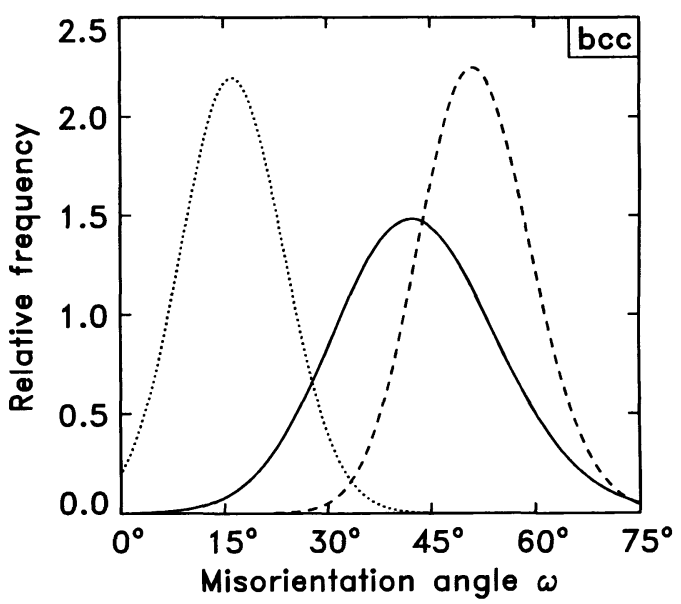

Figure 2 (c) The same situation as in (a) except that we have replaced the cubic grains by 339 Wigner-Seitz cells of a body centred cubic lattice.

other. The Gaussians for fcc- and bcc-clusters show a larger half-width than those for the sc-clusters and the shift of the average values due to extremization of $E$ is not so extreme, especially for the maximization of $E$. In sc-clusters the number of faces per grain is only 6 while for the two other types it is 12 or 14, respectively. For that reason the respective maximum and minimum values of $E$ for the sc-clusters can attain extremer values than for the other types.

The distribution of misorientations depending on the number of grains for the different types of clusters is shown in Figure 3 to 5 . The original uncorrelated distribution is nearly independent of the number of grains (Figure 3). Small deviations, especially for low number of grains, are due to statistical errors. With increasing number of grains the minimization of $E$ results in smaller half-widths and in lower average values for the distributions (Figure 4). If we maximize $E$ the respective effects cannot be recognized (Figure 5). The main reason for this different behaviour is easy to understand. Because of the 3 dimensions of the Euler space the number of misorientations in a certain interval $\Delta \omega$ increases quadratically with $\omega$. As becomes obvious there are a lot of possibilities to realize a misorientation in the order of $\omega=45^{\circ}$, but there are decisively fewer to realize a value of about $\omega=15^{\circ}$. Only for large numbers of grains their arrangement can achieve a large probability of low angles. As a further consequence the original distribution has its maximum value at approximately $45^{\circ}$ and the probability for angles lower than $15^{\circ}$ is nearly zero. Because of the large number of equivalent orientations for materials with cubic crystal symmetry the probability decreases for $\omega>45^{\circ}$.

After these introductory investigations we now examine the effect of orientation correlation on the elastic constants of polycrystalline materials. Materials with isotropic symmetry have only two independent linear elastic constants. In the present work we choose the bulk modulus B and the shear modulus G. For substances with cubic crystal symmetry like copper B is an invariant measure. As a consequence, the bulk modulus is constant, i.e. independent of texture and microstructure. The complete effect of orientation correlation is mirrored into the shear modulus which therefore contains the whole information under study. 


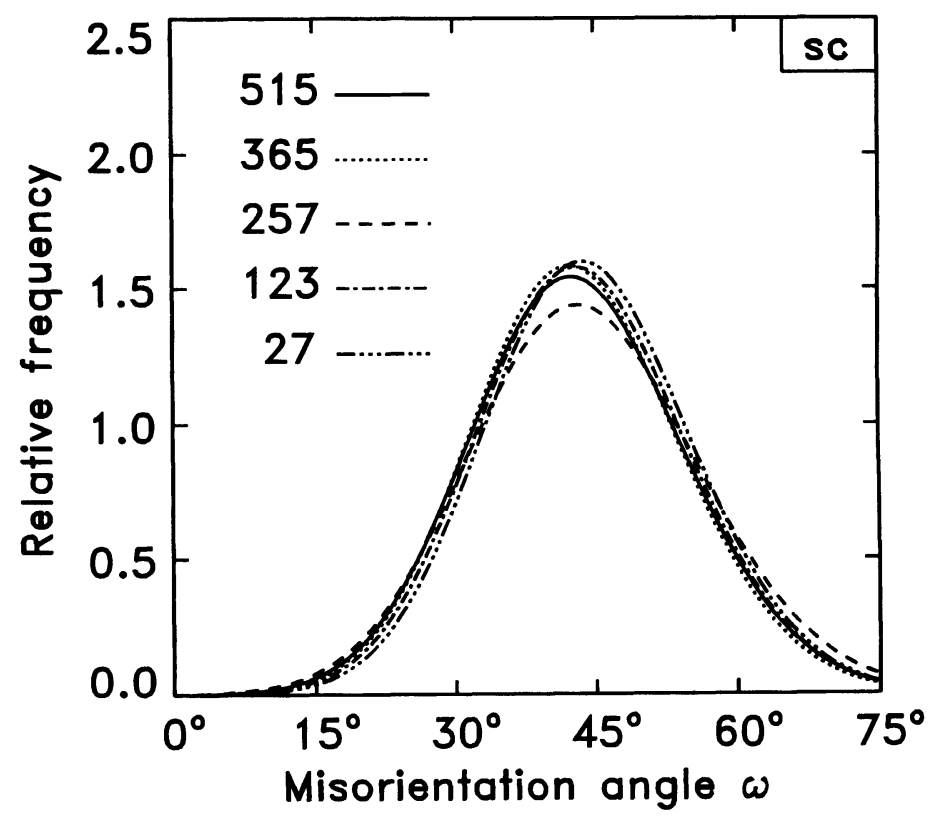

(a)

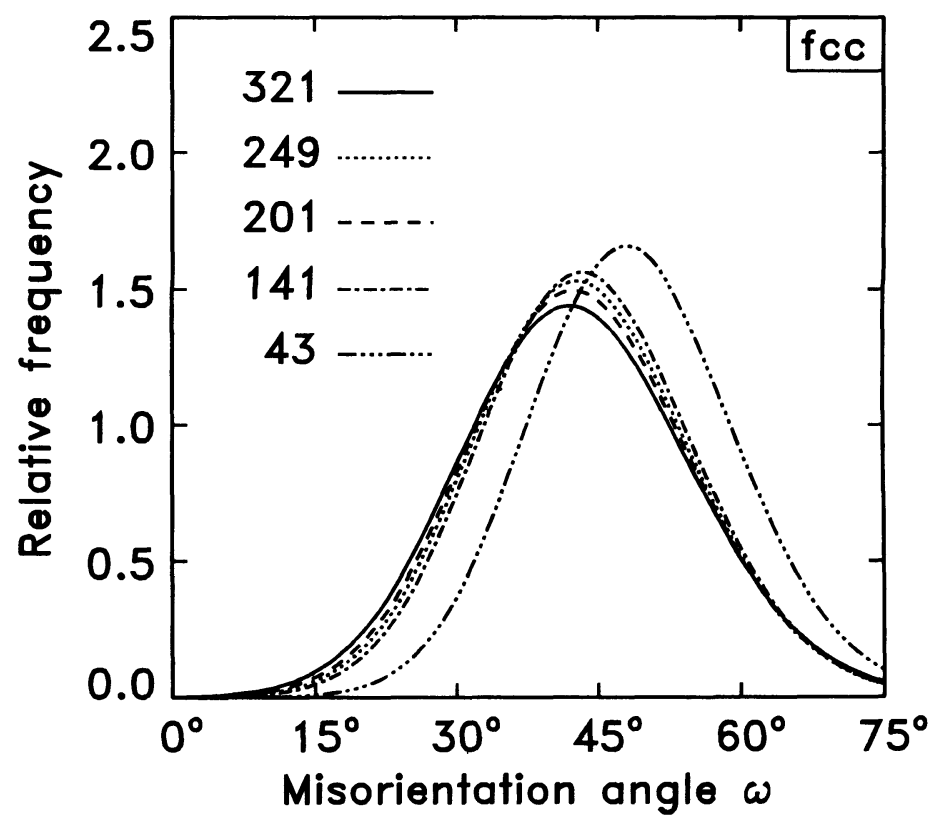

(b)

Figure 3 Distribution of misorientations depending on the number of grains used for the simulations. The orientations of grains are uncorrelated. (a) Results for sc-clusters. (b) Results for fcc-clusters. 


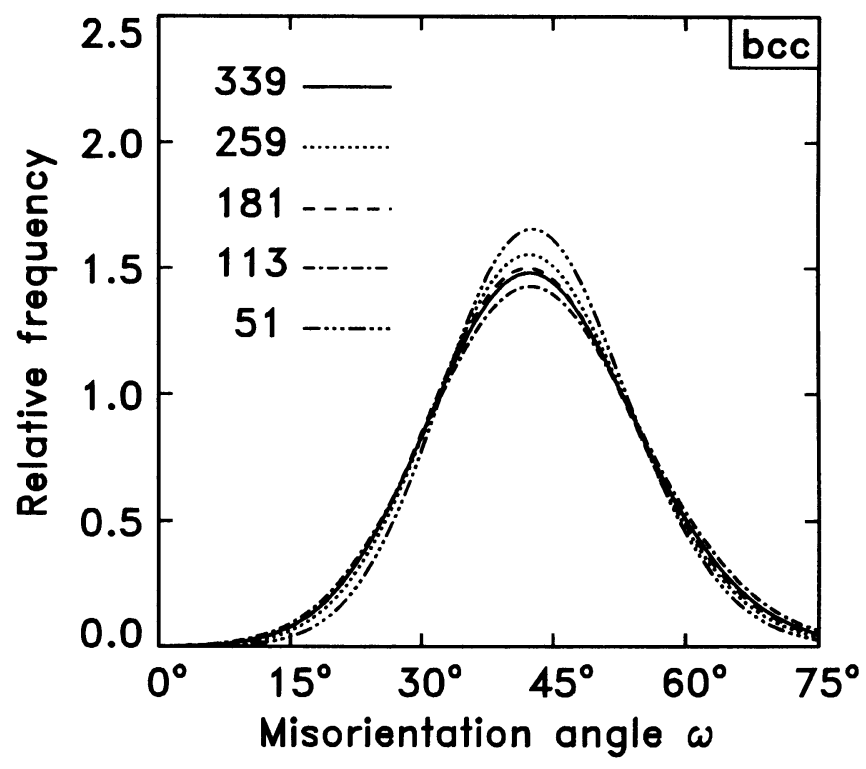

(c)

Figure 3 Distribution of misorientations depending on the number of grains used for the simulations. The orientations of grains are uncorrelated. (c) Results for bcc-clusters.

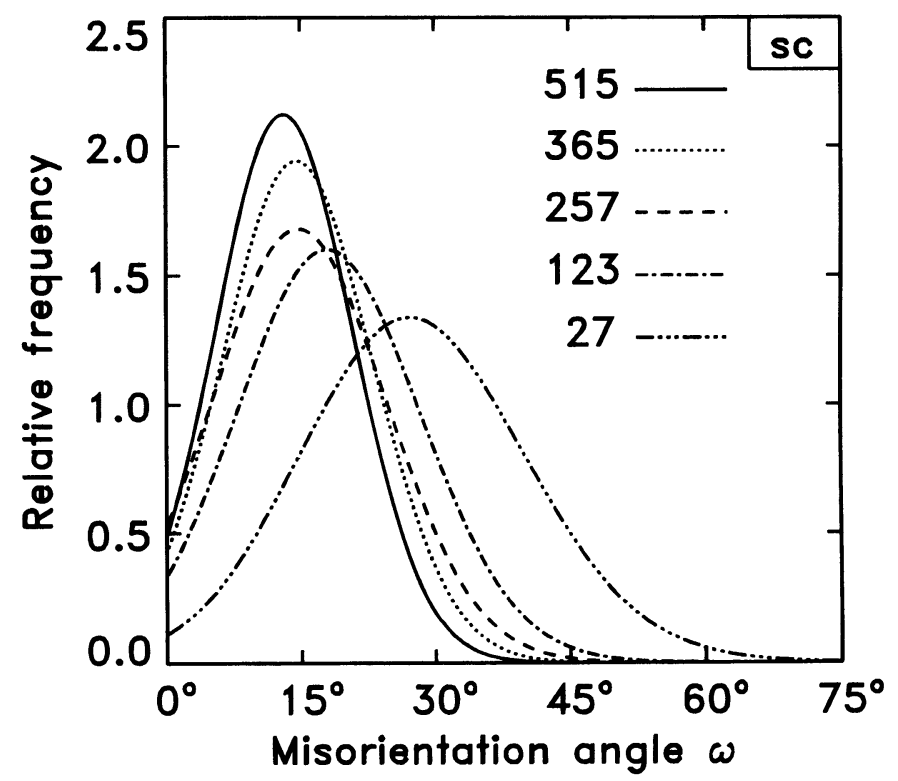

(a)

Figure 4 Distribution of misorientations depending on the number of grains used for the simulations. The orientations of grains were determined by minimizing $\mathrm{E}$ of Eq. (14). (a) Results for sc-clusters. 


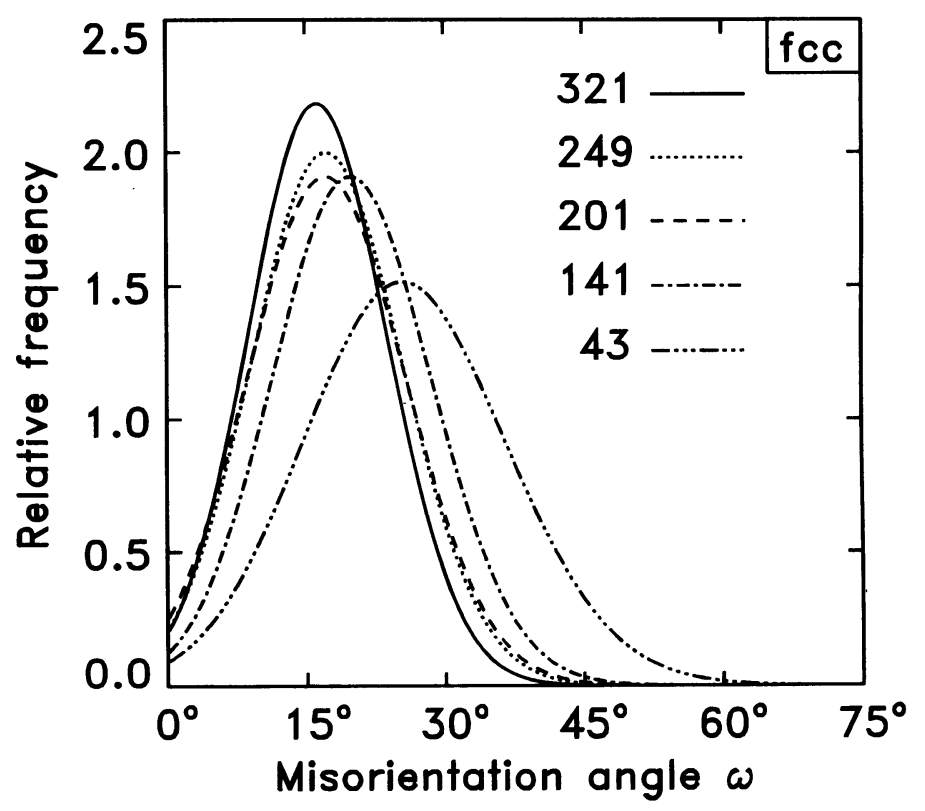

(b)

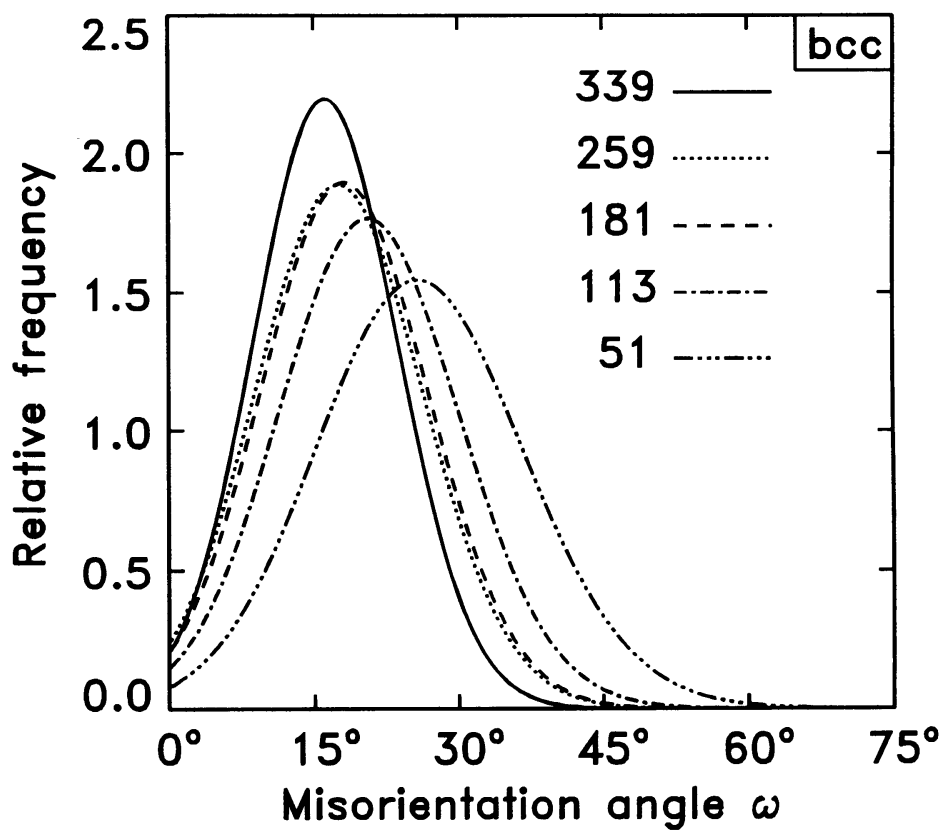

(c)

Figure 4 Distribution of misorientations depending on the number of grains used for the simulations. The orientations of grains were determined by minimizing E of Eq. (14). (b) Results for fcc-clusters. (c) Results for bcc-clusters. 


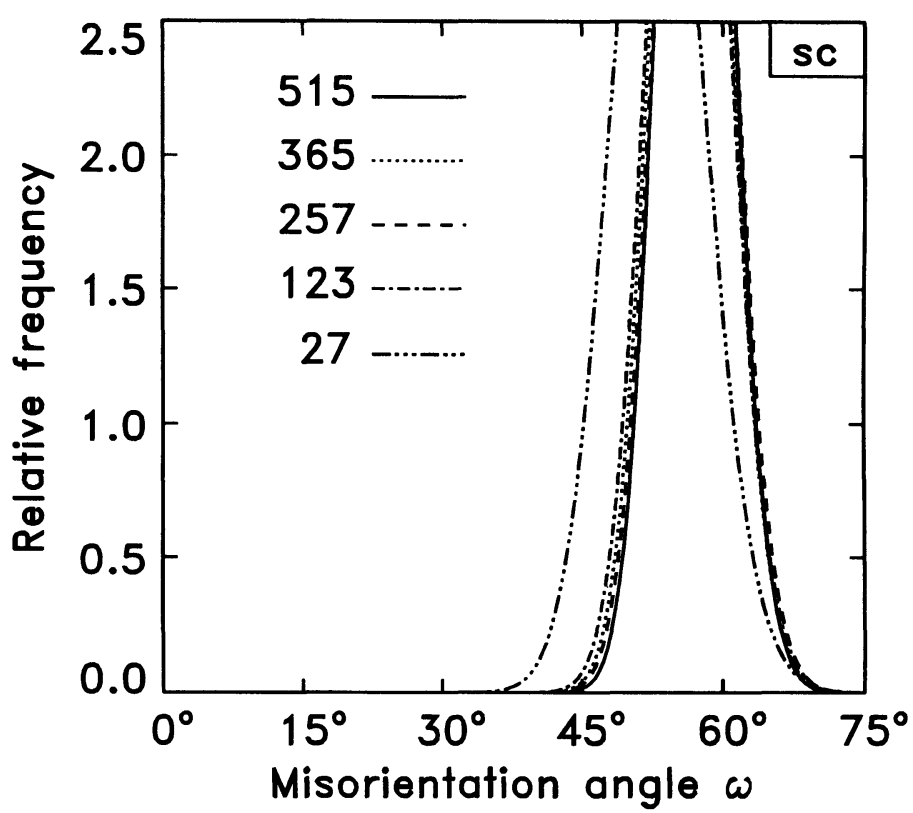

(a)

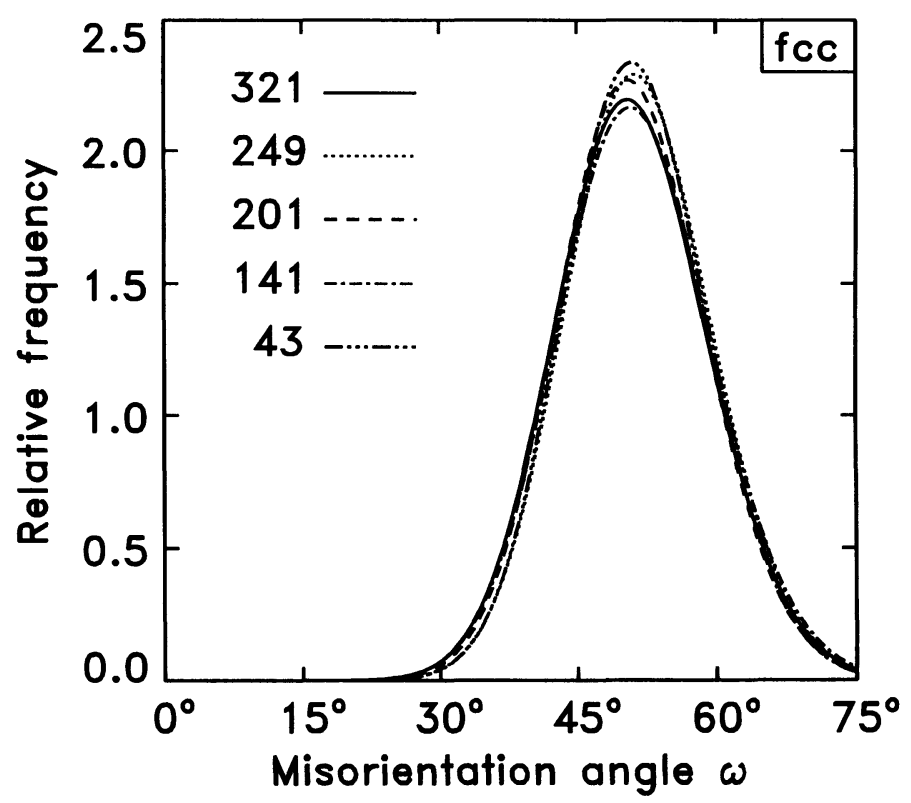

(b)

Figure 5 Distribution of misorientations depending on the number of grains used for the simulations. The orientations of grains were determined by maximizing $E$ of Eq. (14). (a) Results for sc-clusters. (b) Results for fcc-clusters. 


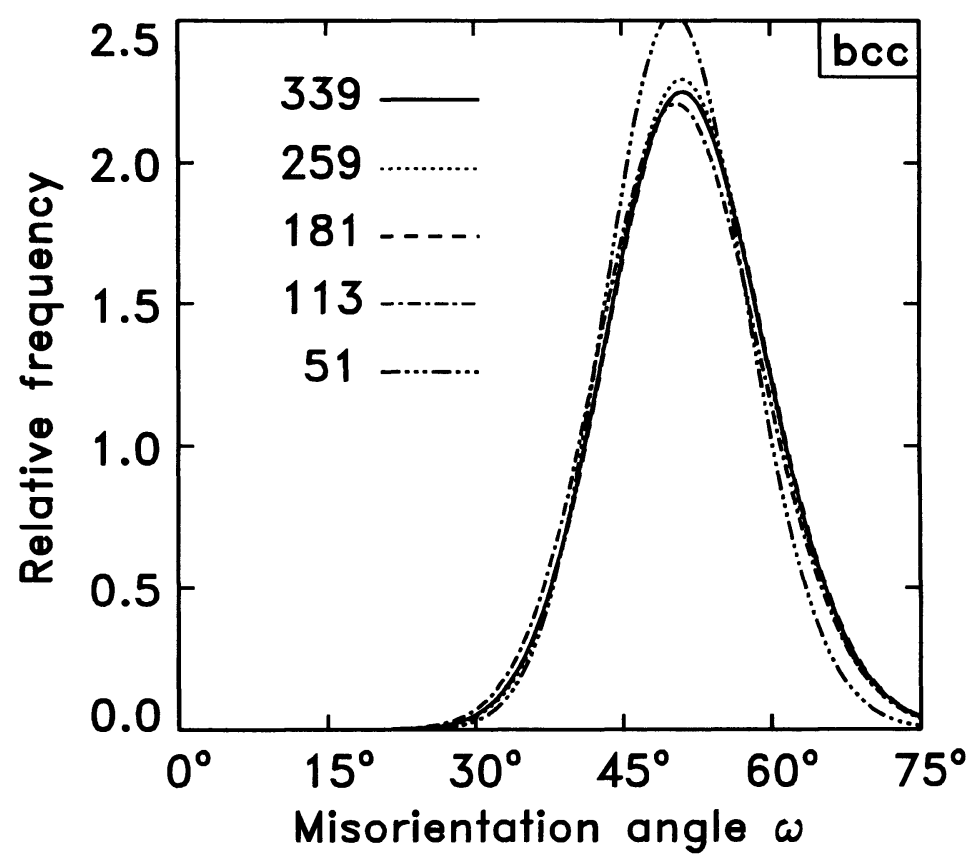

(c)

Figure 5 Distribution of misorientations depending on the number of grains used for the simulations. The orientations of grains were determined by maximizing $E$ of Eq. (14). (c) Results for bcc-clusters.

To get a first estimation we have calculated the well-known values of Voigt and Reuss. All cluster data have to fall within these bounds. Additionally, we have calculated the bounds of Hashin and Shtrikman (1962a, b), which are much closer than those of Hill but are only valid if there is no correlation between neighbouring grains. The latter bounds can serve for comparison with the data obtained with the original uncorrelated distribution. To achieve better statistics all cluster values are averaged over 5 equivalent clusters, i.e. same grain shape and the same criterion for $E$ but another choice of $g_{(\beta)}$.

Figure 6 shows results for the shear modulus for the three different types of clusters depending on their number of grains. As has to be, all moduli fall within the bounds of Hill and the values of the uncorrelated clusters lie always within the bounds of Hashin and Shtrikman. The properties of the distributions Figures 3 to 5 are mirrored in the elastic constants. The values for $G$ of the uncorrelated cluster and those which maximize $E$ are nearly independent of the number of grains. This corresponds to the fact that their Gaussians were independent of the number of grains, too. The shear modulus for the clusters with minimized $E$ increase with increasing number of grains. This effect becomes evident if one regards the respective Gaussians. With increasing number of grains their average value decreases (s. above).

In Figure 7 results for the maximum number of grains are compiled. Two important results become clear. If one maximizes $E$ all moduli decrease by at least $1 \%$. A 


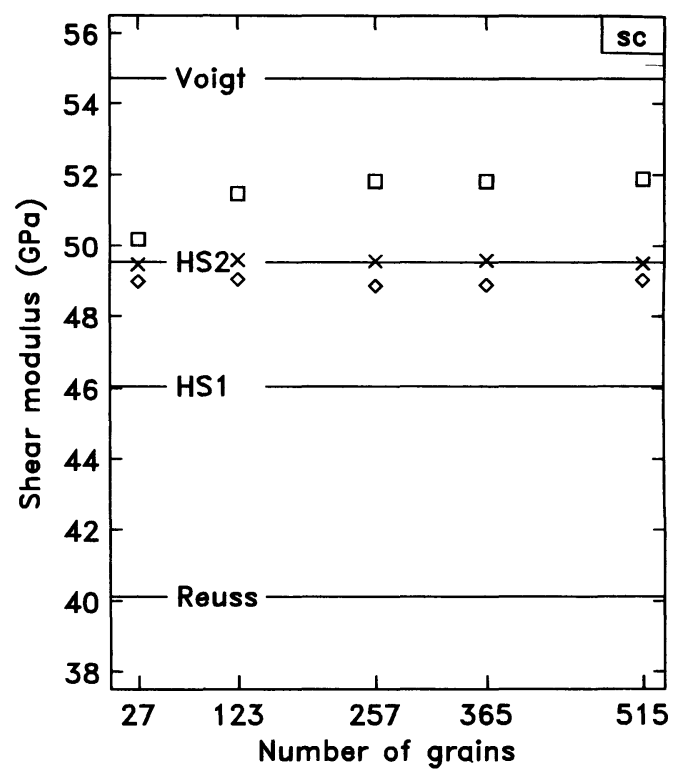

(a)

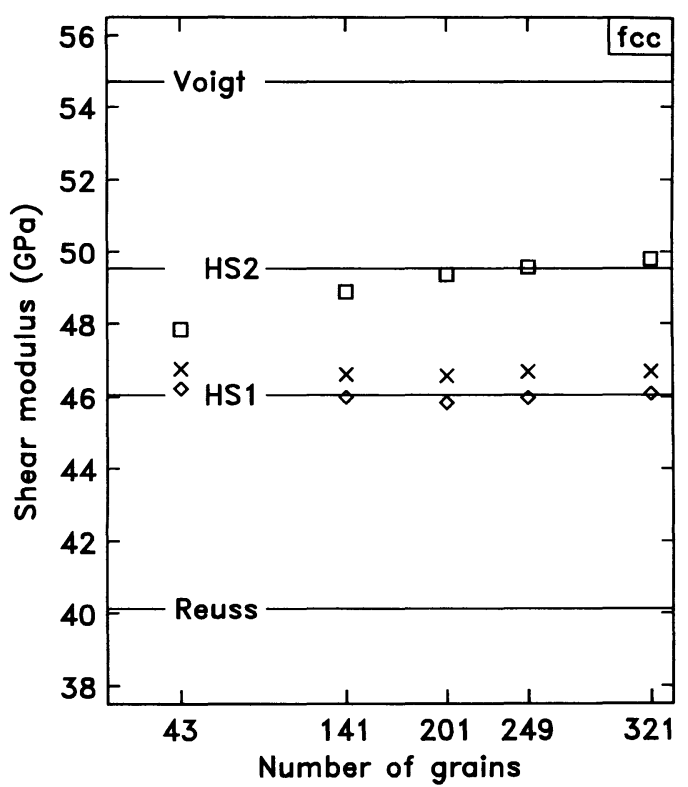

(b)

Figure 6 Shear modulus depending on the number of grains. The crosses denote values for the original distribution of misorientations. If one minimizes $E$ one obtains values displayed by squares. The rhombs mark results for maximizing $E$. This figure corresponds to Figures 3 to 5 . The abbreviations HS1 and HS2 stand for the lower and upper bounds, respectively, of Hashin and Shtrikman. (a) Results for sc-clusters. (b) Results for fcc-clusters. 


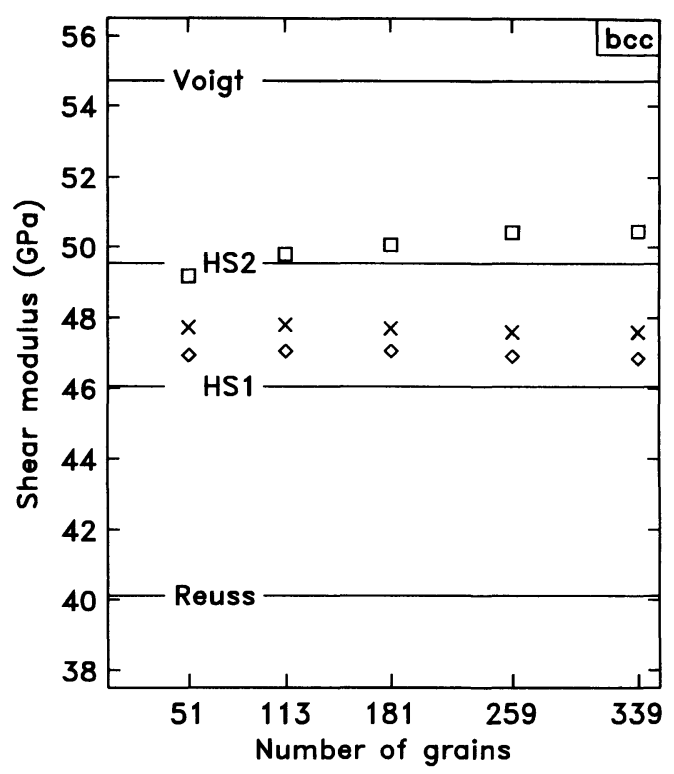

(c)

Figure 6 Shear modulus depending on the number of grains. The crosses denote values for the original distribution of misorientations. If one minimizes $E$ one obtains values displayed by squares. The rhombs mark results for maximizing $E$. This figure corresponds to Figures 3 to 5 . The abbreviations HS1 and HS2 stand for the lower and upper bounds, respectively, of Hashin and Shtrikman. (c) Results for bcc-clusters

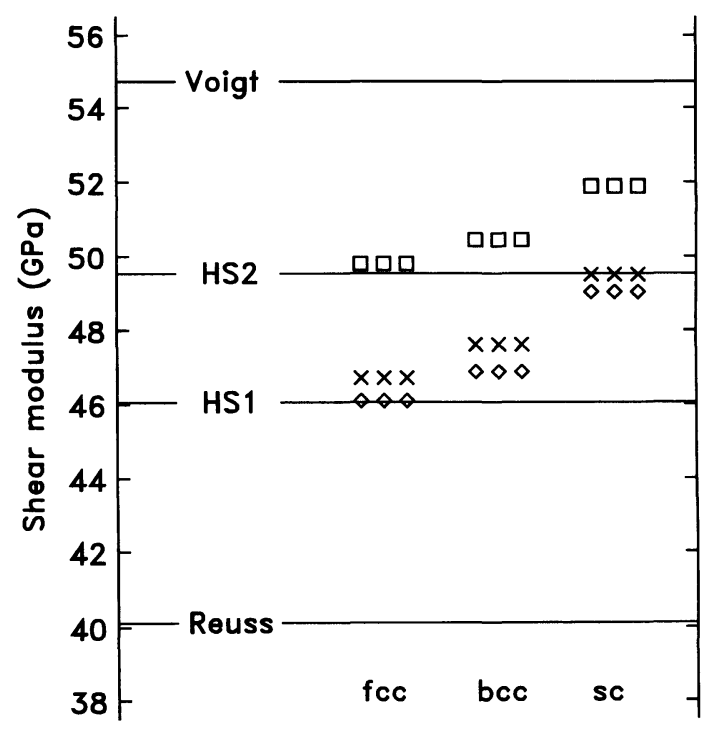

Figure 7 Compilation of results for the shear modulus of Figure 6. Only values obtained for the maximum number of grains for the respective grain shape are displayed. The abbreviations fcc, bcc and sc stand for the type of clusters. 
minimization of $E$ results in an increase of $\mathrm{G}$ up to $6 \%$. This large difference in the change of elastic moduli corresponds to the quite different change of distributions due to extremizing $E$ in opposite directions. The influence of orientation correlation effects is at least as large as the effect of the grain shape on the elastic constants.

\section{CONCLUSIONS}

The present calculations have shown that the applied cluster method is statistically sufficiently significant to detect the influence of orientation correlation across grain boundaries on the macroscopic elastic properties of polycrystalline aggregates. It was found that lower misorientation angles lead to increased elastic stiffness of the aggregate compared with uncorrelated misorientation distribution. Consequently, preferred higher misorientation leads to elastically "softer" behaviour.

The influence of "low-angle correlation" was found to be stronger compared with that of "high-angle correlation". This different behaviour is simply a consequence of the 3-dimensionality of Euler space. Because of the implications of cubic symmetry higher misorientation angles are "mirrored back" to lower ones so that the angle distribution is necessarily limited by the maximum misorientation angle $\omega_{\max }=62.80^{\circ}$ in this symmetry.

The question why "low-angle correlation" leads to "stiffer" material properties cannot yet be conclusively answered. It may, however, be sought in the fact that "low-angle correlation" reaches over a longer distance from any reference grain than uncorrelated arrangement of neighbour orientations. Further calculations will be done to elucidate the problem in more detail.

Former model calculations using the same method have shown that virtually all geometrical parameters of the aggregate have an influence on the macroscopic elastic properties. These were particularly:

- The axis ratios of parallelepipedic grains forming a primitive orthorhombic lattice of cells (grains).

- The shape and with it the number of neighbours of nearly equiaxed grains particularly the Wigner-Seitz cells of the three cubic lattices.

- Even the axis orientation of the cell-lattice can still be recognized in the macroscopic elastic properties.

- Finally in the present paper the influence of orientation correlation was studied. Also this is a "location effect" since the same crystal orientations are only placed in different locations in the aggregate.

The "location effects" are superimposed on the "orientation effects" i.e. the influence of texture on the macroscopic elastic properties. It is seen that "location effects" may fill the margin between the Voigt- and Reuss-bounds. They can definitely exceed the bounds of Hashin-Shtrikman. The difference between the Voigt- and Reuss-bound increases with increasing elastic anisotropy of the crystallites. It becomes zero for vanishing crystal anisotropy. Hence, in this case the influence of the "location parameters" must be zero. 
Orientation parameters i.e. the texture as well as location parameters depend strongly on the history of the material. This applies particularly to the texture, grain size, and grain shape. In the present paper orientation correlation was considered. Also this structural parameter may be different according to the history of the material. In deformed materials orientation correlation of neighbouring cells is assumed according to minimal deformation work. Strong orientation correlations are due to twinning and martensitic transformation. Another type of correlation occurs in grain growth according to minimum grain boundary energy. These correlations can easily be modeled with the method described here.

\section{Acknowledgments}

The present work was financially supported by the Deutsche Forschungsgemeinschaft which is gratefully acknowledged.

\section{References}

Bradley, C. J. and Cracknell, A. P. (1972). The Mathematical Theory of Symmetry in Solids, Clarendon Press, Oxford.

Bunge, H. J. (1982). Texture Analysis in Materials Science, Butterworths, London.

Bunge, H. J. and Weiland, H. (1988). Orientation correlation in grain and phase boundaries, Textures and Microstructures, 7, 231.

Eshelby, J. D. (1957). The determination of the elastic field of an ellipsoidal inclusion, and related problems, Proc. Roy. Soc., A 241, 376.

Hashin, Z. and Shtrikman, S. (1962a). On some variational principles in anisotropic and nonhomogeneous elasticity, J. Mech. Phys. Solids, 10, 335.

Hashin, Z. and Shtrikman, S. (1962b). A variational approach to the theory of the elastic behaviour of polycrystals, J. Mech. Phys. Solids, 10, 343.

Hill, R. (1952). The elastic behaviour of a crystalline aggregate, Proc. Phys. Soc., A 65, 351.

Kiewel, H. and Fritsche, L. (1994). Calculation of effective elastic moduli of polycrystalline materials including non textured samples and fiber textures, Phys. Rev., B 50, 5.

Kiewel, H., Bunge, H. J. and Fritsche, L. (1995). Elastic properties of statistically equivalent materials with varying individual grain orientations, Textures and Microstructures, 25, 17.

Kiewel, H., Bunge, H. J. and Fritsche, L. (1996a). Effect of the grain shape on the elastic constants of polycrystalline material, Textures and Microstructures, in print.

Kiewel, H., Bunge, H. J. and Fritsche, L. (1996b). Estimation of the range of elastic interaction due to grain misorientation, Textures and Microstructures, in print.

Kneer, G. (1964). Zur Elastizität vielkristalliner Aggregate mit und ohne Textur, Doctoral Thesis, Technische Universität Clausthal.

Kneer, G. (1965). Über die Berechnung der Elastizitätsmoduln vielkristalliner Aggregate mit Textur, phys. stat. sol., 9, 825.

Kröner, E. (1958). Berechnung der elastischen Konstanten des Vielkristalls aus den Konstanten der Einkristalle, Z. Phys., 151, 504.

Mackenzie, J. K. (1964). The distribution of rotation axes in random aggregate of cubic crystals, Acta Met., 12, 223.

Morris, P. R. (1971). Iterative scheme for calculating polycrystal elastic constants, Int. J. Eng. Sci., 9, 917.

Reuss, A. (1929). Berechnung der Fließgrenze von Mischkristallen auf Grund der Plastizitätsbedingung für Einkristalle, Z. Angew. Math. Mech., 9, 49.

Voigt, W. (1910). Lehrbuch der Kristallphysik, Teubner, Berlin. 